\title{
Stellar tracers of the Cygnus Arm ${ }^{\star}$ II. A young open cluster in Camelopardalis OB3
}

\author{
I. Negueruela and A. Marco
}

\begin{abstract}
Departamento de Física, Ingeniería de Sistemas y Teoría de la Señal, Escuela Politécnica Superior, Universidad of Alicante, Apdo. 99 , 03080 Alicante, Spain

e-mail: ignacio@dfists.ua.es
\end{abstract}

Received 7 September 2008 / Accepted 10 October 2008

\begin{abstract}
Context. Cam OB3 is the only defined OB association believed to belong to the outer Galactic arm or Cygnus arm. Very few members have been observed and the distance modulus to the association is not well known.

Aims. We attempt a more complete description of the population of Cam OB3 and a better determination of its distance modulus. Methods. We present $u v b y$ photometry of the area surrounding the O-type stars BD $+56^{\circ} 864$ and $\mathrm{LS} \mathrm{I}+57^{\circ} 138$, finding a clear sequence of the early-type stars that define an uncatalogued open cluster, which we call Alicante 1 . We also present spectroscopy of stars in this cluster and the surrounding association.

Results. From the spectral types for 18 very likely members of the association and $U B V$ photometry found in the literature, we derive individual reddenings, finding a extinction law close to standard and an average distance modulus $D M=13.0 \pm 0.4$. This value is in excellent agreement with the distance modulus to the new cluster Alicante 1 found by fitting the photometric sequence to the ZAMS. In spite of the presence of several O-type stars, Alicante 1 is a very sparsely populated open cluster, with an almost total absence of early B-type stars.

Conclusions. Our results definitely confirm that Cam OB3 is located on the Cygnus arm and identify the first open cluster known to belong to the association.
\end{abstract}

Key words. stars: early-type - stars: distances - Galaxy: structure - open clusters and associations: individual: Cam OB3 stars: Hertzsprung-Russell (HR) and C-M diagrams - techniques: photometric

\section{Introduction}

The structure of the Milky Way is still the subject of controversy. While many authors have argued for a four-armed Galaxy (e.g., Vallée 2005; Russeil 2003), two-armed models have also remained popular (e.g., Fernández et al. 2001). Recent results based on star counts from the GLIMPSE survey (Benjamin et al. 2005) favour a two-armed Galaxy, with the Scutum-Crux (i.e., the second arm towards the Galactic centre from the position of the Sun or -II) and the Perseus (the first arm towards the outside from the position of the Sun or $+\mathrm{I}$ ) arms as main features. This is a surprising result, as previous authors had considered the Sagittarius-Carina (-I) and the Norma (-III or internal) arms as the main features (e.g., Georgelin \& Georgelin 1976).

Unfortunately, our view from the Galactic periphery is not the most adequate for studying Galactic structure. For example, the Perseus arm, now believed to be a major spiral feature, cannot be traced beyond Galactic longitude $l \approx 140^{\circ}$, though some tracers seem to be found around $l \approx 180^{\circ}$. This could reflect a flocculent morphology for the outer Milky Way (e.g., Quillen 2002).

Beyond the Perseus arm, molecular clouds definitely delineating an outer arm, generally referred to as the Cygnus arm, are readily visible in $\mathrm{CO}$ surveys all over the first Galactic quadrant (Dame et al. 2001). In most models, the Cygnus arm is

\footnotetext{
* Partially based on observations collected at the 2.2-m telescope (Calar Alto, Spain), the Isaac Newton Telescope (La Palma, Spain) and Observatoire de Haute Provence (CNRS), France.
}

assumed to be the continuation of the Norma arm. In a previous paper (Negueruela \& Marco 2003, henceforth Paper I), we presented spectroscopy for several stars that could belong to the Cygnus arm. Tracers were particularly reliable in the region $l \approx 140^{\circ}-180^{\circ}$, where the Perseus arm is not present along the line of sight. Several stars around $l=147^{\circ}$ were assumed to belong to the association Cam OB3.

The existence of Cam OB3 has sometimes been considered doubtful, as the density of members is not very high and no previously catalogued open clusters are contained. Using data in the literature for 6 likely members, Humphreys (1978) centred it at $\left(l=147^{\circ}, b=+3.0\right)$ and derived $D M=12.6$. Haug (1970) obtained $U B V$ photometry of a larger number of OB stars in the first volume of the Luminous Star catalogue and, based on estimated distances, considered the existence of Cam OB3 certain. In Paper I, we obtained spectroscopy for 10 likely members and found that their calculated distance moduli concentrated very tightly around $D M=13.0$. This $D M$ is one and a half magnitudes higher than those to Per OB1 and Cas OB6, the tracers of the Perseus arm closest in the sky, implying that Cam OB3 is clearly too far away to be on the Perseus arm.

During this work, we noticed that sky images of the area between the O-type stars BD $+56^{\circ} 864$ (O6 Vnn, double-lined spectroscopic binary) and LS I $+57^{\circ} 138(\mathrm{O} 7 \mathrm{~V})$ showed a very high concentration of moderately bright stars. In this paper, we present photometry of the area, clearly showing the presence of an early-type star sequence identifiable as a small open cluster. We also present a rather larger spectroscopic sample of possible 
Table 1. Log of the photometric observations taken at the $2.2-\mathrm{m}$ in October 2002, with all times in seconds.

\begin{tabular}{cccc}
\hline \hline Alicante 1 & $\begin{array}{c}\mathrm{RA}=03 \mathrm{~h} 59 \mathrm{~m} 18.29 \mathrm{~s} \\
(\mathrm{~J} 2000)\end{array}$ & $\begin{array}{c}\text { Dec }=+57^{\circ} 14^{\prime} 13.7^{\prime \prime} \\
(\mathrm{J} 2000)\end{array}$ \\
\hline Filter & Long times & Intermediate times & Short times \\
\hline$u$ & 1400 & 100 & 5 \\
$v$ & 400 & 40 & 2 \\
$b$ & 400 & 40 & 2 \\
$y$ & 400 & 40 & 2 \\
\hline
\end{tabular}

Table 2. Mean catalogue minus transformed values $(D)$ for the standard stars and their standard deviations in $V,(b-y)$ and $c_{1}$.

\begin{tabular}{cccc}
\hline \hline & $V$ & $(b-y)$ & $c_{1}$ \\
\hline$D$ & 0.00 & 0.00 & 0.00 \\
$\sigma$ & 0.02 & 0.03 & 0.03 \\
\hline
\end{tabular}

members of Cam OB3, allowing us a much better definition of the association.

\section{Observations and data}

\subsection{Photometry}

We obtained Strömgren uvby photometry of the region around the star $\mathrm{BD}+56^{\circ} 864$ using BUSCA attached to the 2.2-m telescope at the Calar Alto Observatory (Almeria, Spain) on the nights of 24-26 October 2002.

The instrument splits the light into 4 channels, each equipped with a CCD camera, which can take images at the same time in different filters. We used the 3 bluer channels to obtain images with the uvby filters. Each camera covers a field of view of $12^{\prime} \times$ $12^{\prime}$ and has a pixel scale of 0 ' $^{\prime} 176$. Images from the area were taken using 3 series of different exposure times to obtain accurate photometry for a magnitude range. These exposure times are given in Table 1.

The reduction of the images was done with IRAF $^{1}$ routines for the bias and flat-field corrections. The photometry was obtained by point-spread function (PSF) fitting using the DAOPHOT package (Stetson 1987) provided by IRAF. The apertures were the same for standard and target stars. To construct the PSF empirically, we manually selected bright stars (typically 25 stars) over the whole field as good candidates for PSF stars. Once we had the list of candidate PSF stars, we determined an initial PSF by fitting the best function among the 5 options available in the PSF routine inside DAOPHOT. We chose the PSF to be variable in order 2 across the frame to take the systematic pattern of PSF variability with position on the chip into account.

We selected secondary standard stars from Marco \& Bernabeu (2001). We performed atmospheric extinction corrections and solved the transformation equations following the procedure described in Marco \& Bernabeu (2001). The precision of the photometry was calculated as the dispersion of the mean of the difference (transformed value - catalogue value). The values for this run are given in Table 2.

In Fig. 1, we show the area observed. Likely members of the cluster are numbered. As this is a newly discovered cluster, we

${ }^{1}$ IRAF is distributed by the National Optical Astronomy Observatories, which are operated by the Association of Universities for Research in Astronomy, Inc., under cooperative agreement with the National Science Foundation.

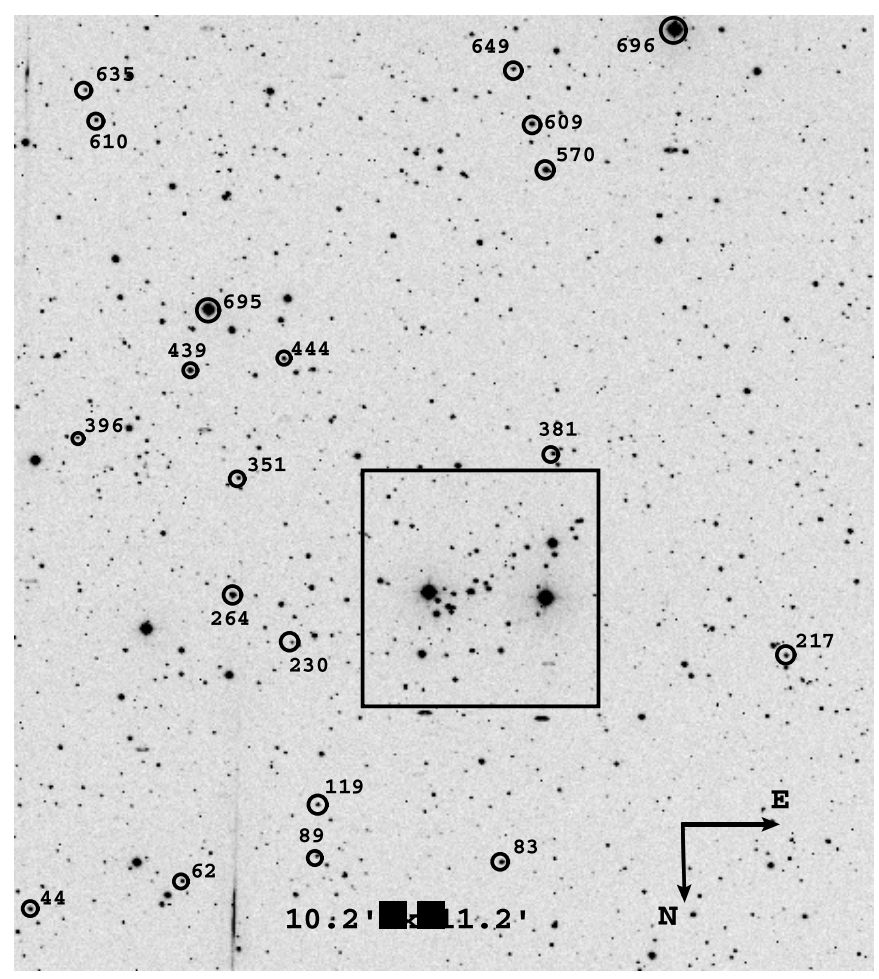

Fig. 1. Finding chart for stars belonging to the blue sequence in Fig. 4 (possible cluster members). $X Y$ positions are listed in Table 7, where they are correlated to RA and Dec. The origin of coordinates is located in the bottom left corner of the image. The area inside the square is displayed in the following figure. North is down and east to the right. This is a $y$-band image taken with BUSCA. The field of view is $10^{\prime} .2 \times$ 11.2 and the central coordinates are RA $=03 \mathrm{~h} 59 \mathrm{~m} 19 \mathrm{~s}$ and Dec $=$ $+57^{\circ} 11^{\prime} 53^{\prime \prime}$ in epoch J2000.

use our own numbering system. In Fig. 2, we zoom in on the area of the image marked with a square, which contains the majority of cluster members. In Table 7, we give $X$ and $Y$ positions for stars in Figs. 1 and 2, together with their identification in the 2MASS catalogue an their coordinates (right ascension, RA, and declination, Dec) in epoch $\mathbf{J} 2000$. In Table 8, we give the values of $V,(b-y)$ and $c_{1}$ for these stars.

\subsection{Spectroscopy}

Observations of several stars in Cam OB3, including the three O-type stars likely connected with the new cluster, were presented in Paper I. These spectra were obtained with the Aurélie spectrograph on the 1.52-m telescope at the Observatoire de Haute Provence (OHP) during runs on 18-22 January 2002 and 25-28 February 2002 (see Paper I for details). The spectra of the three O-type stars in the cluster area are shown in Fig. 3.

A few objects, which were not observed over the whole 3960-4910 A range, were not included in Paper I, as their spectral types were not certain, in most cases because the spectra clearly showed lines corresponding to two stars. These observations are listed in Table 3.

Other spectra of these and other stars in Cam OB3 were obtained with the Intermediate Dispersion Spectrograph (IDS) at the 2.5-m Isaac Newton Telescope (INT) in La Palma (Spain) during a run on 23-26 July 2002. The instrument was equipped with the $235-\mathrm{mm}$ camera, the R1200Y grating, and the Tek\#5 CCD. This configuration gives a dispersion of 


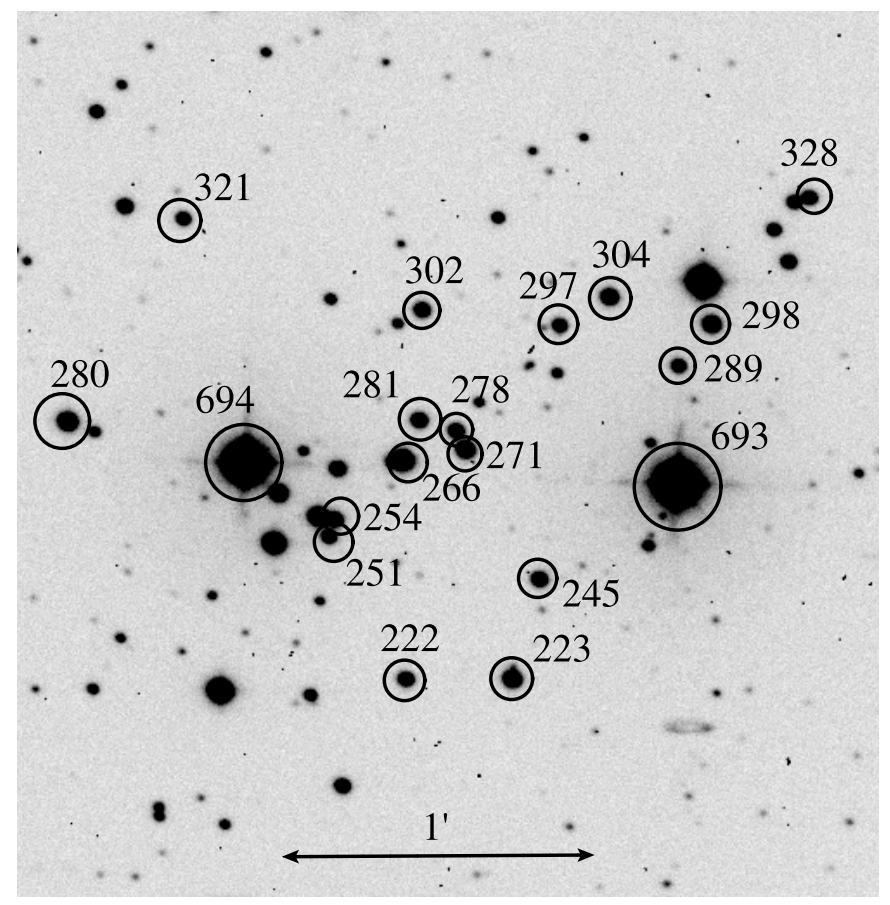

Fig. 2. Finding chart for likely cluster members in the central concentration. $X Y$ positions are listed in Table 7, where they are correlated to RA and Dec. The origin of coordinates is located in the bottom left corner of Fig. 1.

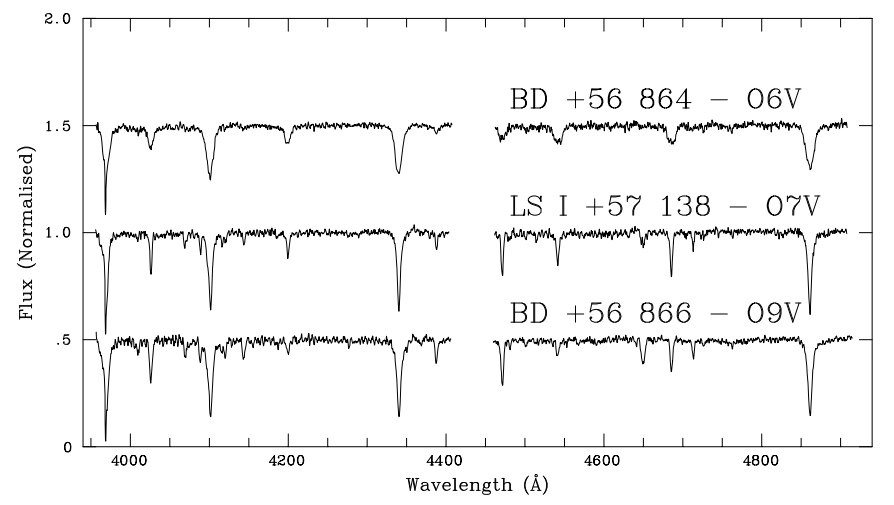

Fig. 3. OHP 1.52-m spectra of the three O-type stars in the area that we investigated. The identifications in Figs. 1 and 2 are $\mathrm{BD}+56^{\circ} 864=$ $\# 693$, LS I $+57^{\circ} 137=\# 695$ and $\mathrm{BD}+56^{\circ} 866=\# 696$. The small gap around $\lambda=4420 \AA$ indicates the division between the two poses. Details of the observations are given in Paper I.

$\sim 0.8 \AA /$ pixel over the 4050-4900 A range. The complete log of these observations is given in Table 4.

Finally, spectra of possible members of the new cluster were obtained during different runs. Some were observed with the INT on 25-28 October 2002. The instrument was equipped with the 235-mm camera and the EEV \#10 CCD. We used the R1200B grating for the brightest members (covering 3900-5200 $\AA$ ) and the R400V grating for faint stars (covering 3500-7200 $\AA$ ). Other members were observed on 29 August 2008 with the 2.6-m Nordic Optical Telescope (La Palma) and ALFOSC. We used grism \#14 to cover the 3900-6800 Å range. Some of these spectra are shown in Figs. 7 and 8.

All the spectroscopic data have been reduced with the Starlink packages CCDPACK (Draper et al. 2000) and FIGARO
Table 3. Observations from the OHP $1.52-\mathrm{m}$ telescope that were not included in Paper I.

\begin{tabular}{lccc}
\hline \hline Name & Date & $\begin{array}{c}\text { Exposure } \\
\text { time }(\mathrm{s})\end{array}$ & $\begin{array}{c}\text { Wavelength } \\
\text { range }\end{array}$ \\
\hline $\mathrm{HD} 237204$ & $28 / 02 / 02$ & 900 & $3960-4410 \AA$ \\
$\mathrm{BD}+55^{\circ} 837$ & $25 / 02 / 02$ & 1500 & $4460-4910 \AA$ \\
$\mathrm{BD}+55^{\circ} 838$ & $25 / 02 / 02$ & 1500 & $4460-4910 \AA$ \\
$\mathrm{LS} \mathrm{I}+57^{\circ} 137$ & $21 / 01 / 02$ & 1800 & $4460-4910 \AA$ \\
$\mathrm{LS} \mathrm{V}+56^{\circ} 59$ & $20 / 01 / 02$ & 1800 & $3960-4410 \AA$ \\
\hline
\end{tabular}

Table 4. Possible members of Cam OB3 observed from the INT in July 2002.

\begin{tabular}{lccc}
\hline \hline $\begin{array}{l}\text { LS } \\
\text { number }\end{array}$ & $\begin{array}{c}\text { Other } \\
\text { name }\end{array}$ & Date & $\begin{array}{c}\text { Exposure } \\
\text { time }(\mathrm{s})\end{array}$ \\
\hline $\mathrm{I}+56^{\circ} 90$ & - & $26 / 07 / 02$ & 800 \\
$\mathrm{I}+56^{\circ} 92$ & - & $23 / 07 / 02$ & 450 \\
$\mathrm{I}+56^{\circ} 94$ & - & $25 / 07 / 02$ & 500 \\
$\mathrm{I}+56^{\circ} 98$ & HD 237204 & $26 / 07 / 02$ & 300 \\
$\mathrm{I}+55^{\circ} 53$ & - & $26 / 07 / 02$ & 800 \\
$\mathrm{I}+55^{\circ} 54$ & - & $25 / 07 / 02$ & 500 \\
$\mathrm{I}+55^{\circ} 55$ & $\mathrm{BD}+55^{\circ} 837$ & $23 / 07 / 02$ & 300 \\
$\mathrm{I}+55^{\circ} 57$ & - & $26 / 07 / 02$ & 900 \\
$\mathrm{I}+55^{\circ} 58$ & $\mathrm{BD}+55^{\circ} 838$ & $23 / 07 / 02$ & 300 \\
$\mathrm{I}+55^{\circ} 47$ & - & $25 / 07 / 02$ & 900 \\
$\mathrm{~V}+56^{\circ} 59$ & - & $25 / 07 / 02$ & 400 \\
$\mathrm{I}+57^{\circ} 136$ & - & $25 / 07 / 02$ & 600 \\
$\mathrm{I}+57^{\circ} 137$ & - & $26 / 07 / 02$ & 720 \\
$\mathrm{I}+57^{\circ} 140$ & - & $26 / 07 / 02$ & 500 \\
\hline
\end{tabular}

(Shortridge et al. 1997) and analysed using FIGARO and DIPSO (Howarth et al. 1997).

\section{Results}

\subsection{Photometry}

We start by plotting the $V /(b-y)$ diagram for all stars in the field (see Fig. 4). The diagram shows evidence of different stellar populations, with at least two well-defined sequences. To the left of the diagram, there is a clear sequence with bluer colours, $(b-y) \lesssim 0.5$. Catalogued OB stars lie at the top of this sequence, so it seems sensible to assume that this sequence corresponds to an associated population. To check this hypothesis, we selected all the stars belonging to this sequence and plot their $V-c_{1}$ diagram (see Fig. 5). The stars also form a clear sequence in this diagram, with colours typical of reddened early type stars. Stars belonging to the sequence are marked in Figs. 1 and 2. It is obvious that the vast majority of the stars in the sequence are grouped together in a small area surrounding the two mid O-type stars. In view of the spatial concentration of the photometric sequence, we conclude that these stars are members of a previously uncatalogued open cluster in this region, which we provisionally call Alicante 1.

Our procedure may leave out some cluster members, but guarantees that most candidates selected are cluster members. The sequence shows little spread in $E(b-y)$ (see following section), making it unlikely that main-sequence members with higher reddenings are left out. Moreover, Zdanavičius et al. (2005) argue that most of the reddening in this direction originates close to the Sun. The young age of Alicante 1 (see Sect. 4.1) precludes the existence of evolved members. Premain-sequence (PMS) members are likely to exist, and our 


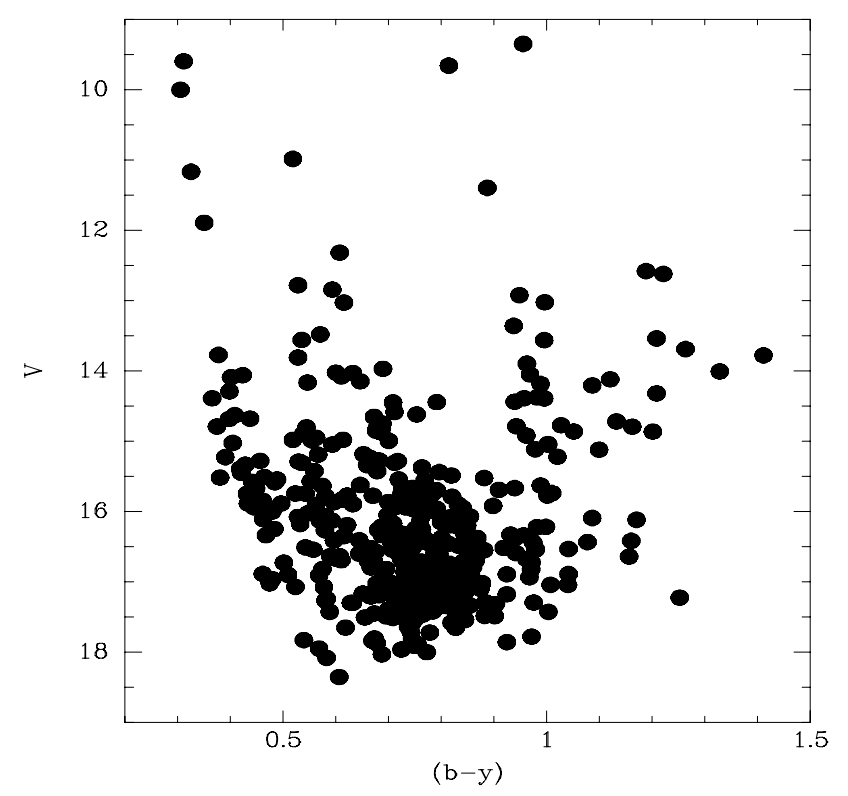

Fig. 4. $V /(b-y)$ diagram for all stars in the field. We identify the bluer sequence as the new cluster Alicante 1.

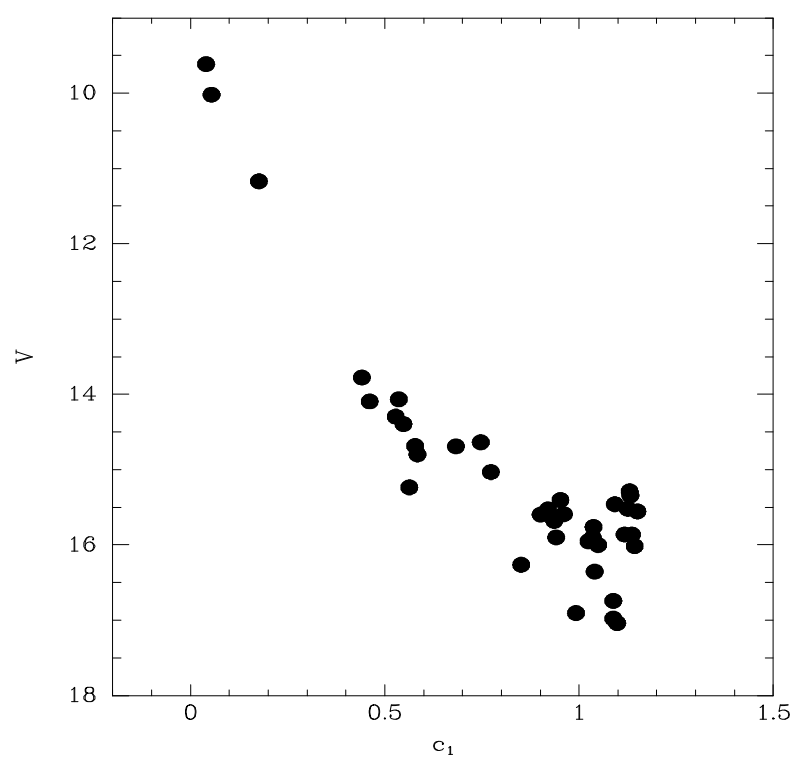

Fig. 5. $V / c_{1}$ diagram for the stars stars identified as possible cluster members in Fig. 4.

selection procedure is likely to leave out most of them. Finding these PMS objects out from photometry alone is very difficult, as they will not have typical colours, and the cluster is sparse and mixed with large foreground and background populations. A search for emission-line objects may produce a sample of this population.

Our selection of candidates leaves 38 stars as possible members of the new cluster Alicante 1. Now we can use the photometric analysis to determine its distance. First, we need to deredden the values for $V,(b-y)$ and $c_{1}$. For this purpose, we calculate individual reddenings following the procedure described by Crawford et al. (1970) for B-type stars: we use the observed $c_{1}$ to predict the first approximation to $(b-y)_{0}$ with the expression $(b-y)_{0}=-0.116+0.097 c_{1}$. Then we calculate $E(b-y)=(b-y)-(b-y)_{0}$ and use $E\left(c_{1}\right)=0.2 E(b-y)$ to correct $c_{1}$ for reddening $c_{0}=c_{1}-E\left(c_{1}\right)$. The intrinsic colour $(b-y)_{0}$ is now calculated by replacing $c_{1}$ with $c_{0}$ in the above equation for $(b-y)_{0}$. Three iterations are enough to reach convergence in the process. The final values of $E(b-y), E\left(c_{1}\right)$ and $c_{0}$ are given in Table 8 . We note that this procedure assumes that the reddening law is the average for nearby stars. We will see in Sect. 3.3 that this assumption is justified.

The reddening is quite constant across the cluster. The average value is $E(b-y)=0.48$ for 38 possible members, with a dispersion of only $\sigma=0.03$, comparable to the precision of the $(b-y)$ colour in our photometry. No star deviates by more than $2 \sigma$ from the average value, which corresponds to $E(B-V)=0.67$.

Next, we plot the $V_{0} /(b-y)_{0}$ and $V_{0} / c_{0}$ diagrams (see Fig. 6) and calculate the distance modulus $D M$ that gives the best fit to the ZAMS (Perry et al. 1987) in both diagrams. The best fit for the distance modulus is $D M=13.0 \pm 0.2$, using the sequence of late $\mathrm{B}$ and early-A stars. In the $V_{0} / c_{0}$ diagram, it is clear that the three brightest members are displaced vertically from their expected positions. This is a suggestion of a binary nature, and we see in Sect. 3.2.1 that two of the three are spectroscopic binaries. Their position in the diagram is compatible with the same $D M$ if this is taken into account. In Table 8, we list the dereddened photometric indices and the derived values for absolute magnitudes calculated as $M_{V}=V_{0}-13.0$.

\subsection{Spectroscopy}

\subsubsection{Cluster members}

The brightest member of Alicante 1 is BD $+56^{\circ} 864$ (\#693). Several spectra of this object show double lines (see Fig. 7). The two components have similar spectral types around O6 V. This system is a short period binary and will be studied in detail in forthcoming work. Double lines are also evident in the OHP spectrum of LS I $+57^{\circ} 137$ (\#695). The secondary shows weaker metallic lines and therefore appears to be slightly later than the primary. The INT spectrum can be classified as B1.5 V, but, given the double lines, this may be a combination of a $\sim \mathrm{B} 1 \mathrm{~V}$ and $\mathrm{a} \sim \mathrm{B} 2 \mathrm{~V}$ star.

The expected absolute magnitude of an $06 \mathrm{~V}$ star is $M_{V}=$ -4.9 (Martins et al. 2005), so the observed $M_{V}=-5.4$ of $\mathrm{BD}+56^{\circ} 864$ is compatible with the presence of a secondary almost as bright as the primary. The expected magnitude of a B1 V star is $M_{V}=-3.2$, and again the observed $M_{V}=-3.7$ of LSI $+57^{\circ} 137$ is compatible with the presence of a bright secondary. The spectrum of LS I $+57^{\circ} 138$ (\#694) (O7 V), the second brightest member, does not show any evidence of a secondary component (see Fig. 3). The observed $M_{V}=-5.0$ is also a bit too high for a single star, but within the expected dispersion of the calibration $\left(M_{V}=-4.6\right.$ according to Martins et al. 2005).

To the south of the field lies BD $+56^{\circ} 866$. Though this star is marked in Fig. 1 as \#696, it is too close to the edge of the chip, and we could not obtain its photometry. However, its published photometry clearly shows it to be a member of Cam OB3 (see Table 5), so it is almost certainly a cluster member. The $V /(b-y)$ diagram (Fig. 4) shows a star with $V \approx 12$ that seems to belong to the cluster sequence, but is not present in Fig. 5 . This object was very close to the edge of the chip in the short $u$ exposure, and we have no $c_{1}$ index for it. It is identified as GSC 03725-00486, and its 2MASS colours show it to be an early-type star, though its $\left(J-K_{\mathrm{S}}\right)$ is smaller than those of cluster members. We took a spectrum of this object, which turns out to be an A giant, hence not a cluster member. 

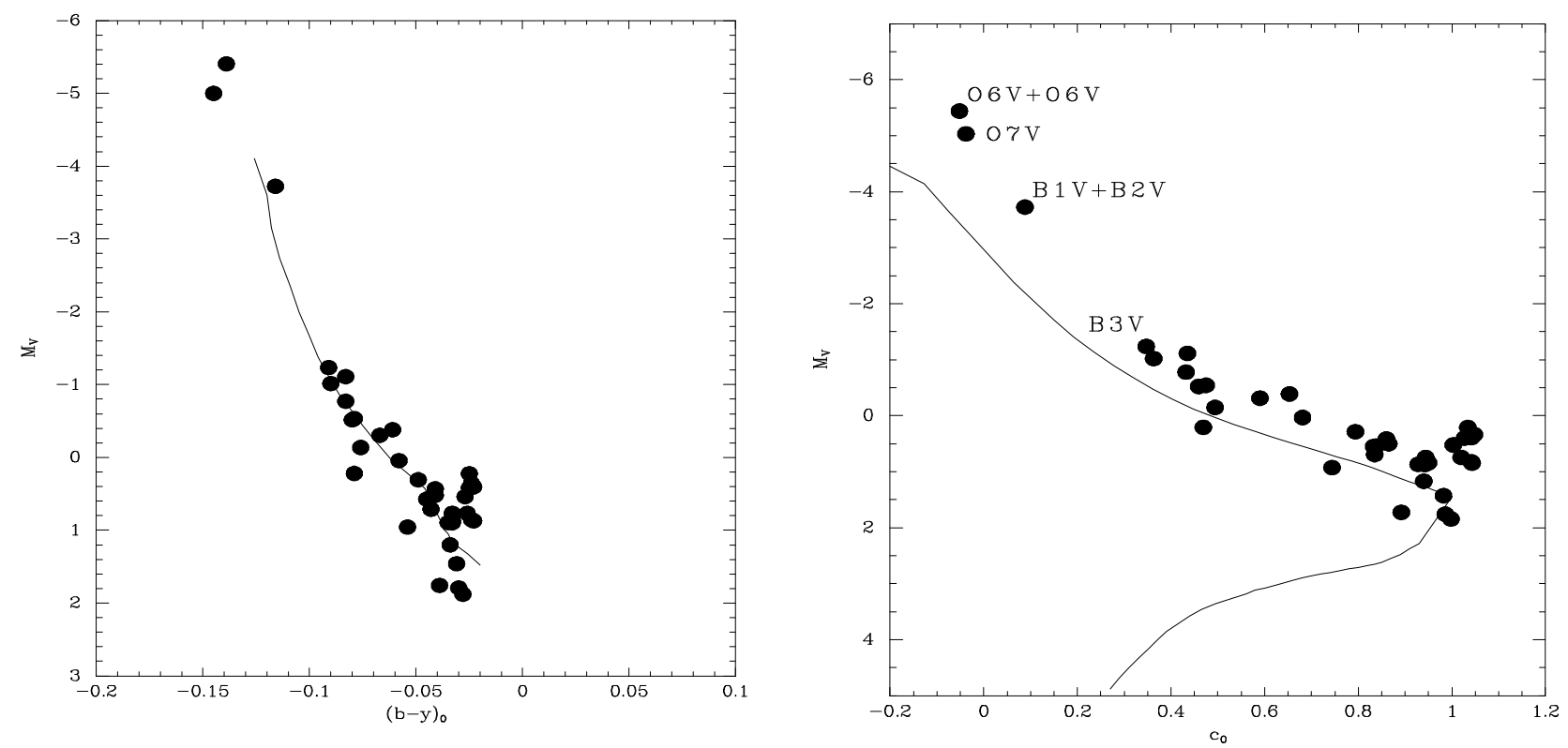

Fig. 6. Left: dereddened $M_{V} /(b-y)_{0}$ diagram for cluster members. Right: dereddened $M_{V} / c_{0}$ diagram for members. The spectral types for the brightest members are indicated. In both panels, the line represents the ZAMS from Perry et al. (1987).

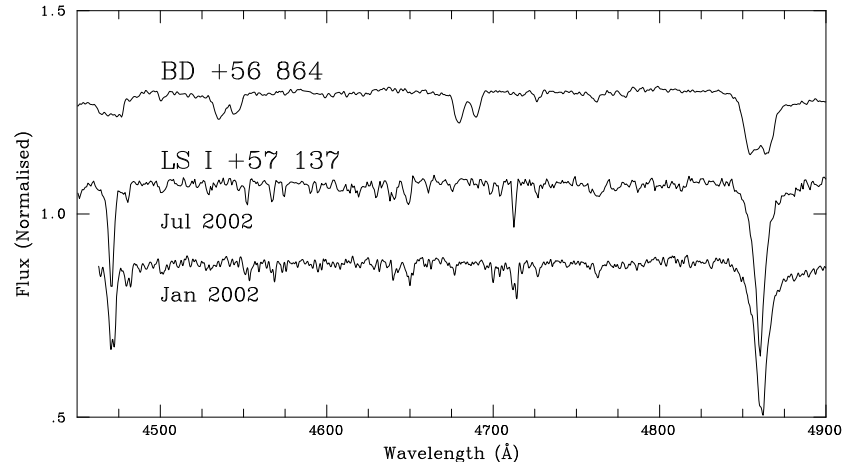

Fig. 7. Spectra of two of the brightest members of Alicante 1 showing that they are double-lined spectroscopic binaries. BD $+56^{\circ} 864$, originally classified as $\mathrm{O} 6 \mathrm{Vnn}$, has two similar components. In the case of LS I $+57^{\circ} 137$, the spectrum from 2002 July shows a single component of spectral type B1.5 V. However, the 2002 January spectrum clearly shows double He I and Mg I 4481 Å lines and weak second components in other metallic lines.

Figure 8 shows the spectra of other likely members. The brightest non-catalogued member, \#266, has spectral type B3 V. The next brightest stars, \#264 and \#439, have spectral types B4-B5 V, in very good agreement with their positions in the photometric diagrams. Other stars observed by chance are \#289 and \#278. Star \#289 is located in the cluster core. It has weak Ca II $3933 \AA$, compatible with the purely interstellar line seen in the B-type stars. The prominent Si II $4128 \AA$ doublet then suggests that this is a chemically peculiar $\mathrm{Bp}(\mathrm{Si})$ star. The spectral type is $\sim \mathrm{B} 9$, and the object is likely to be a Bp member. Star \#278 is also in the cluster core. It has a spectral type around A1 III-IV. As its reddening is typical of cluster members and its position in the $M_{V} / c_{0}$ diagram is compatible with the same distance modulus as the rest of the cluster, it may only be a chance alignment of a nearby unrelated star or a PMS cluster member. PMS stars without emission lines have been identified in clusters with low background contamination, such as NGC 1893 (Negueruela et al. 2007). As a matter of fact, \#278 is part of

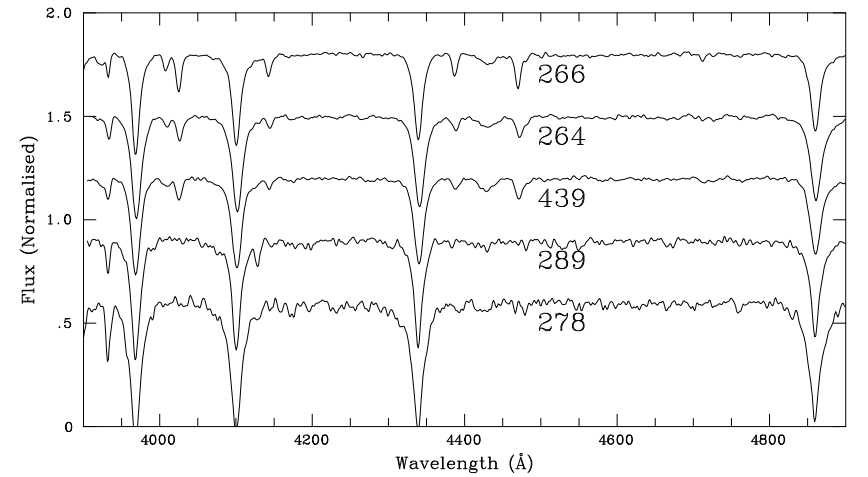

Fig. 8. Spectra of some cluster members. Stars \#264, \#266, and \#439 are the brightest members in the photometric sequence after the catalogued OB stars, Stars \#289 and \#278 are fainter members observed by chance. See text for the spectral classifications.

a small clump of objects with $c_{0} \approx 1.0$, lying slightly above the rest of the sequence (Fig. 6), and this discussion may apply to all of them.

\subsubsection{Other members of Cam OB3}

For all the candidate members of Cam OB3, we derive spectral types following the prescriptions described in Paper I. The resulting spectral types are listed in Table 5. We use this information to derive the reddening and distance to the stars in the next section. In the OHP spectrum, LS V $+56^{\circ} 59$ clearly appears as a double-lined spectroscopic binary. The H I and He I lines of the secondary spectrum are weaker than those of the primary, while the metallic lines appear similar in strength. HD 237204 is also likely to be a double-lined spectroscopic binary, as all metallic lines appear very broad in the two available spectra. Rubin (1965), however, did not detect any variability in its radial velocity. The lines of $\mathrm{LS} \mathrm{I}+57^{\circ} 140$ are also broad and asymmetric, but the presence of a secondary spectrum is not certain. 
Table 5. Candidate members of Cam OB3, with their spectral types, photometry from the literature, and derived parameters.

\begin{tabular}{|c|c|c|c|c|c|c|c|c|c|}
\hline $\begin{array}{l}\text { LS } \\
\text { number }\end{array}$ & $l$ & $b$ & $\begin{array}{l}\text { Spectral } \\
\text { type }\end{array}$ & $\bar{V}$ & Ref..$^{(1)}$ & $R$ & $E(B-V)$ & $m_{V}$ & $D M$ \\
\hline $\mathrm{I}+55^{\circ} 47$ & $143^{\circ} .5$ & -1.5 & O9 III & 11.25 & $\mathrm{~h}$ & $2.88 \pm 0.08$ & $1.35 \pm 0.03$ & $7.34 \pm 0.04$ & 12.6 \\
\hline $\mathrm{I}+56^{\circ} 90$ & $145^{\circ} .9$ & +1.9 & $\mathrm{~B} 1.5 \mathrm{~V}(+)$ & 12.24 & $\mathrm{~h}$ & $2.74 \pm 0.12$ & $0.81 \pm 0.03$ & $9.98 \pm 0.04$ & 12.8 \\
\hline $\mathrm{I}+57^{\circ} 136$ & $146^{\circ} 0$ & +2.9 & $\mathrm{O} 8.5 \mathrm{~V}$ & 10.88 & $\mathrm{~h}$ & $2.92 \pm 0.15$ & $0.66 \pm 0.03$ & $8.90 \pm 0.04$ & 13.1 \\
\hline $\mathrm{I}+56^{\circ} 92$ & $146^{\circ} 0$ & +2.4 & B5 IIe & 10.26 & $\mathrm{~h}$ & $3.38 \pm 0.14$ & $0.86 \pm 0.03$ & $7.31 \pm 0.04$ & 12.7 \\
\hline $\mathrm{I}+57^{\circ} 140$ & $146^{\circ} .1$ & +3.5 & $\mathrm{~B} 0.2 \mathrm{IV}(+)$ & 11.02 & $\mathrm{~h}$ & $2.91 \pm 0.17$ & $0.60 \pm 0.03$ & $9.22 \pm 0.04$ & 13.7 \\
\hline$I+56^{\circ} 94$ & $146^{\circ} 2$ & +2.8 & B1.5 IV & 11.77 & $\mathrm{~h}$ & $3.00 \pm 0.25$ & $0.46 \pm 0.03$ & $10.37 \pm 0.04$ & 13.7 \\
\hline $\mathrm{I}+57^{\circ} 137$ & 146.3 & +3.1 & $\mathrm{~B} 1.5 \mathrm{~V}+$ & 11.23 & $\mathrm{~h}$ & $2.90 \pm 0.20$ & $0.52 \pm 0.03$ & $9.66 \pm 0.04$ & 12.5 \\
\hline $\mathrm{I}+57^{\circ} 138$ & $146^{\circ} 3$ & $+3: 1$ & $\mathrm{O} 7 \mathrm{~V}$ & 10.09 & $\mathrm{~h}$ & $3.20 \pm 0.19$ & $0.56 \pm 0.02$ & $8.27 \pm 0.04$ & 12.9 \\
\hline $\mathrm{I}+57^{\circ} 139$ & $146^{\circ} 3$ & +3.1 & $\mathrm{O} 6 \mathrm{~V}+$ & 9.67 & $\mathrm{~h}$ & $2.74 \pm 0.18$ & $0.56 \pm 0.02$ & $8.14 \pm 0.04$ & 13.1 \\
\hline$I+56^{\circ} 97$ & $146^{\circ} 4$ & +3.1 & O9 V & 10.33 & $\mathrm{~h}$ & $2.94 \pm 0.17$ & $0.64 \pm 0.03$ & $8.42 \pm 0.04$ & 12.5 \\
\hline $\mathrm{I}+56^{\circ} 98$ & $146^{\circ} 6$ & +3.0 & B0.5 V+ & 9.17 & $\mathrm{~h}$ & $2.86 \pm 0.19$ & $0.54 \pm 0.03$ & $7.59 \pm 0.04$ & 11.1 \\
\hline $\mathrm{I}+55^{\circ} 54$ & $146^{\circ} 9$ & +1.8 & B0.2 IV & 11.77 & $\mathrm{~h}$ & $2.92 \pm 0.12$ & $0.85 \pm 0.03$ & $9.26 \pm 0.04$ & 13.8 \\
\hline$I+55^{\circ} 53$ & $147^{\circ} .7$ & +1.5 & B3 III* & 11.83 & $\mathrm{~h}$ & $3.11 \pm 0.11$ & $1.00 \pm 0.03$ & $8.69 \pm 0.04$ & 11.7 \\
\hline $\mathrm{I}+55^{\circ} 55$ & $147^{\circ} 0$ & +2.0 & BC1.5 Ib & 9.59 & $\mathrm{~h}$ & $2.97 \pm 0.13$ & $0.88 \pm 0.03$ & $6.94 \pm 0.04$ & 12.7 \\
\hline $\mathrm{I}+56^{\circ} 99$ & $147^{\circ} .1$ & +3.0 & O9.5 Iab & 9.00 & $\mathrm{~h}$ & $3.13 \pm 0.14$ & $0.74 \pm 0.02$ & $6.64 \pm 0.04$ & 12.9 \\
\hline$V+56^{\circ} 56$ & $147^{\circ} .4$ & +3.9 & B5 Ia & 7.99 & hi & $3.51 \pm 0.19$ & $0.64 \pm 0.03$ & $5.73 \pm 0.04$ & 12.9 \\
\hline$I+55^{\circ} 57$ & 147.5 & +1.8 & B1 IV* & 12.19 & $\mathrm{~h}$ & $2.96 \pm 0.14$ & $0.78 \pm 0.03$ & $9.81 \pm 0.04$ & 13.6 \\
\hline $\mathrm{I}+55^{\circ} 58$ & 147.6 & +1.9 & B2.5 Iab & 9.29 & $\mathrm{~h}$ & $2.98 \pm 0.11$ & $1.00 \pm 0.03$ & $6.28 \pm 0.04$ & 12.8 \\
\hline $\mathrm{V}+55^{\circ} 11$ & $147^{\circ} .6$ & +2.7 & B6 Ia & 8.72 & hi & $3.15 \pm 0.14$ & $0.84 \pm 0.03$ & $6.04 \pm 0.04$ & 13.0 \\
\hline $\mathrm{V}+56^{\circ} 59$ & $149^{\circ} .7$ & +5.3 & $\mathrm{~B} 1 \mathrm{~V}+$ & $10.92^{*}$ & - & - & - & - & 12.6 \\
\hline $\mathrm{V}+56^{\circ} 60$ & $149^{\circ} .8$ & +5.7 & B2.5 III & $11.51^{*}$ & - & - & - & - & 13.7 \\
\hline $\mathrm{V}+53^{\circ} 22$ & $151^{\circ} .6$ & +2.9 & B0 Ia & 9.32 & hi & $3.21 \pm 0.13$ & $0.90 \pm 0.03$ & $6.39 \pm 0.04$ & 13.0 \\
\hline
\end{tabular}

(1) The references for the $U B V$ photometry are (h) Haug (1970) or (hi) Hiltner (1956). The $J H K_{\mathrm{S}}$ photometry is from 2MASS. Two stars have no accurate $U B V$ photometry and their distance moduli are estimates (see Paper I).

\subsection{Reddening and distance to Cam OB3}

To determine the reddening and distance to the isolated stars, we used $U B V$ photometry from the literature. As in Paper I, we took Haug (1970) as our prime source of photometry, supplemented by Hiltner (1956). Both authors use the same photometric system. In addition, we collected $J H K_{\mathrm{S}}$ photometry from the 2MASS catalogue (Skrutskie et al. 2006). The photometry was used as input for the $\chi^{2}$ code for parametrised modelling and characterisation of the photometry and spectroscopy CHORIZOS implemented by Maíz-Apellániz (2004). This code generates synthetic photometry from a stellar model and convolves it with extinction laws from Cardelli et al. (1989). A fit to the real photometric data determines the values of $R$ and $E(B-V)$ that would most likely result in the observed photometry for a star with the given spectrum. In Table 5, we list the spectral types derived for all candidate members of Cam OB3, the photometry collected from the literature and the values obtained for $R$ and $E(B-V)$. We consider all the candidate members from Paper I and the objects listed in Table 4.

The results of the fits with CHORIZOS favour values of $R$ very close to the standard value $R=3.1$. There are few exceptions. LS I $+56^{\circ} 92$ is an emission-line object (cf. Negueruela 2004) and the high value of $R$ found reflects the presence of a circumstellar excess. The value adopted for the $D M$ of this object is an approximation assuming standard reddening and no excess in $E(B-V)$ (formally, this should be taken as a lower limit, but is unlikely to differ much from the actual value). The B5 Ia supergiant HD 25914 (LS V +56 56) also has a very high value for $R$, suggesting a circumstellar excess as well. This is a known variable (GQ Cam), likely to be a very luminous star. Leaving aside these two objects, we have an average $R=2.97 \pm 0.14$ (where the error represents the standard deviation of the sample). This value is compatible within errors with the standard value. Based on a photometric study of stars in the area, Zdanavičius et al. (2005) conclude that $R=2.9$, in very good agreement with
Table 6. Absolute magnitude calibration used here.

\begin{tabular}{lcccccc}
\hline \hline & V & IV & III & II & Ib & Ia \\
\hline O6 & -4.9 & - & -5.7 & - & - & -6.4 \\
O7 & -4.6 & - & -5.5 & - & - & -6.5 \\
O8 & -4.3 & - & -5.4 & - & - & -6.5 \\
O9 & -4.1 & - & -5.3 & - & -6.0 & -6.5 \\
O9.5/7 & -3.9 & - & -5.2 & - & -6.0 & -6.6 \\
B0 & -3.8 & -4.5 & -5.1 & -5.8 & -6.0 & -6.6 \\
B0.2 & -3.8 & -4.5 & -5.1 & -5.6 & -6.0 & -6.7 \\
B0.5 & -3.5 & -4.2 & -5.0 & -5.5 & -6.0 & -6.9 \\
B0.7 & -3.5 & -4.2 & -4.8 & -5.3 & -6.0 & -7.0 \\
B1 & -3.2 & -3.8 & -4.3 & -5.1 & -6.0 & -7.0 \\
B1.5 & -2.8 & -3.3 & -3.9 & -5.0 & -5.8 & -7.2 \\
B2 & -2.5 & -3.1 & -3.7 & -4.8 & -5.8 & -7.4 \\
B2.5 & -2.0 & -3.1 & -3.5 & -4.8 & -5.8 & -7.4 \\
B3 & -1.6 & -2.5 & -3.0 & -4.7 & -5.8 & -7.2 \\
\hline
\end{tabular}

our value, and that extinction is caused mostly by material at $<1.5 \mathrm{kpc}$ from the Sun. This result justifies the assumption of standard reddening used when dereddening the Strömgren photometry in Sect. 3.1. We note a tendency for double-lined stars to give lower values of $R$, though there are too few such systems to know if this is a real trend.

With these values, we calculated the distance modulus to each object. Since the publication of Paper I, several works have advocated a reduction in the temperature scale and absolute magnitude scale of O-type stars. Because of this, we used the absolute magnitude scale of Martins et al. (2005) rather than the one used in Paper I. For B spectral types, the calibration of Humphreys \& McElroy (1984) was used. In the B0-B1 interval, the calibrations are not consistent, and an interpolation has been made, resulting in the calibration given in Table 6 . For the two luminosity class Iab supergiants in the sample, we adopt $M_{V}=-6.3$ for LS I $+56^{\circ} 99$ (=HD 237211, O9.5 Iab) and 
Table 7. Coordinates $(X Y)$ in the map (Fig. 1) of likely members of Alicante 1 with ubvy photometry, including 2MASS identification for these stars and their coordinates.

\begin{tabular}{|c|c|c|c|c|c|}
\hline Number & RA (J2000) & $\overline{\text { Dec (J2000) }}$ & Name (2MASS) & $\bar{~} X$ (Pixels) & 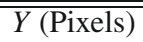 \\
\hline $695^{*}$ & 035846.53 & +571043.6 & $059.693873+57.178764$ & 414.93 & 1411.15 \\
\hline 266 & 035911.36 & +571410.5 & $059.797345+57.236237$ & 975.86 & 808.75 \\
\hline 264 & 035849.39 & +571417.2 & $059.705783+57.238117$ & 466.86 & 801.75 \\
\hline 570 & 035917.33 & +570852.9 & $059.822194+57.148026$ & 1135.31 & 1708.00 \\
\hline 439 & 035844.97 & +571129.6 & $059.687384+57.191555$ & 376.07 & 1280.98 \\
\hline 280 & 035903.00 & +571404.2 & $059.762506+57.234509$ & 782.23 & 831.13 \\
\hline 609 & 035915.96 & +570818.4 & $059.816485+57.138458$ & 1106.08 & 1806.73 \\
\hline 271 & 035940.68 & +571452.1 & $059.919501+57.247795$ & 1012.12 & 814.95 \\
\hline 223 & 035914.29 & +571454.2 & $059.809535+57.248375$ & 1039.09 & 682.36 \\
\hline 298 & 035919.05 & +571341.0 & $059.829361+57.228069$ & 1154.34 & 887.31 \\
\hline 304 & 035916.47 & +57 1336.1 & $059.818637+57.226681$ & 1095.23 & 903.19 \\
\hline 44 & 035831.32 & +571816.2 & $059.630503+57.304493$ & 34.41 & 131.62 \\
\hline 83 & 035914.86 & +571732.4 & $059.811910+57.292332$ & 1040.82 & 231.82 \\
\hline 281 & 035911.80 & +571402.1 & $059.799157+57.233925$ & 985.28 & 831.91 \\
\hline 245 & 035914.89 & +571433.8 & $059.812057+57.242710$ & 1054.73 & 740.04 \\
\hline 302 & 035911.80 & +571339.7 & $059.799169+57.227707$ & 986.99 & 895.67 \\
\hline 278 & 035912.72 & +571404.1 & $059.803008+57.234467$ & 1006.64 & 825.77 \\
\hline 254 & 035914.89 & +571433.8 & $059.812057+57.242710$ & 936.50 & 774.56 \\
\hline 217 & 035940.68 & +571452.1 & $059.919501+57.247795$ & 1648.13 & 672.37 \\
\hline 381 & 035918.66 & +571225.4 & $059.827744+57.207047$ & 1150.71 & 1103.12 \\
\hline 444 & 035853.60 & +571119.3 & $059.723338+57.188694$ & 576.28 & 1305.51 \\
\hline 222 & 035911.62 & +571454.8 & $059.798424+57.248566$ & 977.58 & 682.26 \\
\hline 328 & 035921.02 & +571315.9 & $059.837604+57.221096$ & 1210.75 & 960.55 \\
\hline 251 & 035909.61 & +571426.2 & $059.790049+57.240608$ & 933.08 & 764.68 \\
\hline 289 & 035918.25 & +571349.7 & $059.826022+57.230476$ & 1135.10 & 863.31 \\
\hline 649 & 035914.10 & +570737.4 & $059.808753+57.127056$ & 1065.84 & 1924.29 \\
\hline 297 & 035915.23 & +571342.2 & $059.813468+57.228401$ & 1066.39 & 886.79 \\
\hline 321 & 035905.77 & +571322.5 & $059.774047+57.222904$ & 848.99 & 948.38 \\
\hline 351 & 035849.63 & +571249.2 & $059.706797+57.213665$ & 478.45 & 1051.91 \\
\hline 610 & 035835.77 & +5708 23.5 & $059.649042+57.139870$ & 175.46 & 1815.13 \\
\hline 62 & 035845.35 & +571752.4 & $059.688969+57.297894$ & 359.40 & 191.55 \\
\hline 119 & 035857.73 & +571653.0 & $059.740534+57.281399$ & 648.78 & 353.67 \\
\hline 89 & 035857.74 & +571731.5 & $059.740576+57.292084$ & 646.15 & 244.04 \\
\hline 635 & 035834.70 & +570801.3 & $059.644580+57.133690$ & 152.05 & 1879.19 \\
\hline 230 & 035854.97 & +571451.8 & $059.729049+57.247719$ & 593.33 & 700.30 \\
\hline 396 & 035834.71 & +571222.2 & $059.644608+57.206169$ & 135.28 & 1136.67 \\
\hline $694^{*}$ & 035907.49 & +571411.7 & $059.781192+57.236591$ & 883.50 & 806.50 \\
\hline 693* & 035918.30 & +571413.8 & $059.826231+57.237160$ & 1134.50 & 795.50 \\
\hline
\end{tabular}

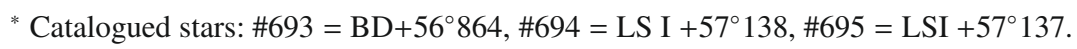

$M_{V}=-6.5$ for $\mathrm{LS} \mathrm{I}+55^{\circ} 58\left(=\mathrm{BD}+55^{\circ} 838\right.$, B2.5 Iab $)$. The values obtained are also listed in Table 5.

With these values, HD 237204 (LS I $+56^{\circ} 98$ ) is clearly a foreground object at a distance compatible with the Perseus arm. The other 19 objects give $D M=12.9 \pm 0.5(1 \sigma)$. LS I $+55^{\circ} 53$ has a distance modulus $>2 \sigma$ shorter than the average, and its spectral type (B3 III) suggests a star significantly less massive than all the other ones. Therefore we also take it for a foreground object. Leaving out this object, we find $D M=13.0 \pm 0.4$.

\section{Discussion}

We found the small cluster Alicante 1 around $\mathrm{BD}+56^{\circ} 864$ and derived a distance modulus $D M=13.0 \pm 0.2$. This value is identical within the errors to the average spectroscopic distance modulus to bright members of Cam OB3. In view of this, we can firmly identify Alicante 1 as the first known cluster in Cam OB3 and confirm the distance modulus to Cam OB3 as 13.0, corresponding to $4.0 \mathrm{kpc}$.

\subsection{Alicante 1}

As there are no evolved stars in Alicante 1, its age cannot be accurately determined. However, the presence of mid O-type stars close to the ZAMS forces it to be $<4$ Myr. On the other hand, the lack of any H II region associated with the stars suggests that they must have had time to disperse their maternal cloud entirely. Therefore an age of $2-3 \mathrm{Myr}$ is favoured. At this age, all stars later than $\sim \mathrm{B} 8\left(\sim 3 M_{\odot}\right)$ must still be in the pre-main-sequence phase.

The HR diagram of Alicante 1 (see Fig. 6) shows an obvious deficiency of early B-type stars. Taking into account the binary nature of $\mathrm{BD}+56^{\circ} 864$ and the very likely membership of $\mathrm{BD}+56^{\circ} 866$, there are at least four O-type stars in the cluster, three more massive than $30 M_{\odot}$. In contrast, the only early B-type stars are the two components of LS I $+57^{\circ} 137$. The photometric sequence only starts at B $3 \mathrm{~V}$ and there are not many B-type members. This mass distribution is quite different from a standard IMF (Kroupa 2001). It may be argued that, in such a small cluster, small number statistics may result in large deviations from a standard value. However, it is tempting to 
Table 8. Photometry of the stars with numbers in Fig. 1.

\begin{tabular}{|c|c|c|c|c|c|c|c|}
\hline Number & $\bar{V}$ & $(b-y)$ & $c_{1}$ & $c_{0}$ & $\overline{M_{V}}$ & $E(b-y)$ & $E\left(c_{1}\right)$ \\
\hline 695 & 11.171 & 0.325 & 0.176 & 0.088 & -3.723 & 0.441 & 0.088 \\
\hline 266 & 13.777 & 0.377 & 0.441 & 0.347 & -1.235 & 0.468 & 0.094 \\
\hline 264 & 14.066 & 0.423 & 0.536 & 0.435 & -1.110 & 0.506 & 0.101 \\
\hline 570 & 14.094 & 0.401 & 0.461 & 0.363 & -1.016 & 0.491 & 0.098 \\
\hline 439 & 14.295 & 0.398 & 0.528 & 0.432 & -0.773 & 0.481 & 0.096 \\
\hline 280 & 14.394 & 0.365 & 0.548 & 0.459 & -0.518 & 0.445 & 0.089 \\
\hline 609 & 14.635 & 0.408 & 0.747 & 0.653 & -0.383 & 0.469 & 0.094 \\
\hline 271 & 14.684 & 0.437 & 0.578 & 0.475 & -0.537 & 0.516 & 0.103 \\
\hline 223 & 14.689 & 0.397 & 0.683 & 0.590 & -0.307 & 0.464 & 0.093 \\
\hline 298 & 14.796 & 0.374 & 0.584 & 0.494 & -0.140 & 0.450 & 0.090 \\
\hline 304 & 15.029 & 0.404 & 0.773 & 0.681 & 0.040 & 0.462 & 0.092 \\
\hline 44 & 15.232 & 0.390 & 0.563 & 0.469 & 0.215 & 0.469 & 0.094 \\
\hline 83 & 15.284 & 0.456 & 1.130 & 1.034 & 0.218 & 0.481 & 0.096 \\
\hline 281 & 15.339 & 0.428 & 1.132 & 1.042 & 0.399 & 0.451 & 0.090 \\
\hline 245 & 15.399 & 0.418 & 0.952 & 0.860 & 0.425 & 0.459 & 0.092 \\
\hline 302 & 15.454 & 0.420 & 1.092 & 1.003 & 0.532 & 0.447 & 0.089 \\
\hline 278 & 15.514 & 0.464 & 1.125 & 1.027 & 0.410 & 0.489 & 0.098 \\
\hline 254 & 15.523 & 0.380 & 0.920 & 0.835 & 0.705 & 0.423 & 0.085 \\
\hline 217 & 15.549 & 0.488 & 1.150 & 1.048 & 0.349 & 0.512 & 0.102 \\
\hline 381 & 15.585 & 0.441 & 0.961 & 0.865 & 0.513 & 0.482 & 0.096 \\
\hline 444 & 15.590 & 0.484 & 0.901 & 0.794 & 0.299 & 0.533 & 0.107 \\
\hline 222 & 15.674 & 0.448 & 0.936 & 0.838 & 0.559 & 0.492 & 0.098 \\
\hline 328 & 15.753 & 0.431 & 1.037 & 0.944 & 0.758 & 0.464 & 0.093 \\
\hline 251 & 15.853 & 0.461 & 1.117 & 1.020 & 0.759 & 0.487 & 0.097 \\
\hline 289 & 15.854 & 0.440 & 1.136 & 1.043 & 0.862 & 0.463 & 0.093 \\
\hline 649 & 15.891 & 0.496 & 0.941 & 0.833 & 0.564 & 0.541 & 0.108 \\
\hline 297 & 15.892 & 0.433 & 1.035 & 0.942 & 0.887 & 0.466 & 0.093 \\
\hline 321 & 15.943 & 0.444 & 1.024 & 0.928 & 0.885 & 0.479 & 0.096 \\
\hline 351 & 15.991 & 0.463 & 1.049 & 0.950 & 0.858 & 0.496 & 0.099 \\
\hline 610 & 16.005 & 0.479 & 1.143 & 1.042 & 0.842 & 0.503 & 0.101 \\
\hline 62 & 16.253 & 0.483 & 0.851 & 0.744 & 0.945 & 0.537 & 0.107 \\
\hline 119 & 16.343 & 0.467 & 1.040 & 0.940 & 1.189 & 0.501 & 0.100 \\
\hline 89 & 16.732 & 0.501 & 1.088 & 0.982 & 1.447 & 0.532 & 0.106 \\
\hline 635 & 16.893 & 0.461 & 0.992 & 0.892 & 1.745 & 0.500 & 0.100 \\
\hline 230 & 16.966 & 0.479 & 1.088 & 0.986 & 1.779 & 0.509 & 0.102 \\
\hline 396 & 17.026 & 0.474 & 1.098 & 0.998 & 1.866 & 0.502 & 0.100 \\
\hline 694 & 10.006 & 0.305 & 0.054 & -0.038 & -5.012 & 0.450 & 0.115 \\
\hline 693 & 9.600 & 0.311 & 0.040 & -0.052 & -5.418 & 0.450 & 0.100 \\
\hline
\end{tabular}

assume that dynamical ejection from the cluster core has played a role in shaping the observed IMF. Clusters containing hard binaries with two components of similar mass may be quite effective at ejecting stars via dynamical ejections (Leonard \& Duncan 1990), especially if massive stars are mainly born as part of multiple systems (Pflamm-Altenburg \& Kroupa 2006). The majority of stars ejected will be B-type stars and their ejection velocity will be inversely proportional to their mass.

The presence of $\mathrm{BD}+56^{\circ} 866 \sim 5^{\prime}$ to the south of the two other O-type stars may represent evidence in favour of ejection, though the stellar distribution in Alicante 1 may instead result from a spread of star formation in small clumps. The only other unevolved O-type star in Cam OB3 is LS I $+57^{\circ} 136(\mathrm{O} 8.5 \mathrm{~V})$, which lies $\sim 20^{\prime}$ away from Alicante 1 . At $d=4.0 \mathrm{kpc}$, this corresponds to a projected separation of $\approx 23$ pc. With a typical runaway speed of $10 \mathrm{~km} \mathrm{~s}^{-1}$, a star would need $2.5 \mathrm{Myr}$ to cover this distance, so a hypothetical ejection of LS I $+57^{\circ} 136$ from Alicante 1 is not unreasonable. Unfortunately, there are no accurate measurements of proper motions for this star.

The region with a higher member density is moderately small, $\sim 2^{\prime}$ across, which corresponds to $\approx 2.3 \mathrm{pc}$ at $d=4.0 \mathrm{kpc}$. It contains 20 possible members earlier than $\sim \mathrm{A} 2$. It is difficult to assess whether the stars around the core represent a halo or rather small groups that have formed in the vicinity of the small cluster.
This spatial distribution, with small concentrations of early-type stars extended over a moderately large area, is not uncommon in this area of the sky (cf. the discussion of clumps of H II regions towards the Anticentre in Moffat et al. 1979).

\subsection{Cam OB3 and the Cygnus arm}

The majority of the members of Cam OB3 listed in Table 5 are evolved massive stars and hence older than Alicante 1. The cluster is not the nuclear region of the association, but may simply represent the latest small star-forming region to have actively created high-mass stars. No other concentrations are obvious amongst members.

In their photometric study of this area, Zdanavičius et al. (2005) failed to find any evidence of the Perseus arm, either as an increase in extinction or a concentration of stars. The lack of intervening material allows the identification of Cygnus arm tracers. Cam OB3 is not a very massive association, but with a radial extent $>100 \mathrm{pc}$ and a significant number of massive stars, it is a reliable spiral tracer. Nearby, Moffat et al. (1979) estimate $D M=13.2 \pm 0.2$ for the young open cluster Waterloo 1 , at $l=151^{\circ} .4$, in very good agreement with the distance to Cam OB3. Moffat et al. (1979) also estimate distances for two small groups of young stars associated with the H II regions Sh 2-217 and Sh 2-219 (at $l \approx 159^{\circ} .3$ ), both of which contain embedded clusters (Deharveng et al. 2003), finding $D M=13.1 \pm$ 0.3 and $D M=13.6 \pm 0.3$, respectively. Therefore it seems that the Cygnus arm is well traced in this area (see discussion in Paper I). The young clusters observed in this area are not very massive, and there seems to be a strong tendency towards sparse groupings of massive stars, rather than compact concentrations.

\section{Conclusions}

Our study of Cam OB3 has resulted in 18 likely members from the Luminous Star catalogue. When their reddenings are calculated individually, they support a value of $R=3.0$, in good agreement with the photometric determination of Zdanavičius et al. (2005). Their average distance modulus is $13.0 \pm 0.4$, in good agreement with previous determinations based on fewer stars.

Around the earliest members of the association, BD $+56^{\circ} 864$ $(\mathrm{O} 6 \mathrm{~V})$ and $\mathrm{LS} \mathrm{I}+57^{\circ} 138(\mathrm{O} 7 \mathrm{~V})$, we find a concentration of B and early A stars, which clearly trace the main sequence of a small and sparse cluster. The sequence also includes the nearby $\mathrm{LS} \mathrm{I}+57^{\circ} 137(\mathrm{~B} 1.5 \mathrm{~V})$ and $\mathrm{BD}+56^{\circ} 866(\mathrm{O} 9 \mathrm{~V})$ and some other faint stars around them. We spectroscopically confirm the brightest uncatalogued members of the sequence to be B3-5 V stars. We call this uncatalogued cluster Alicante 1 and find a distance modulus of $13.0 \pm 0.2$ from ZAMS fitting.

These results definitely confirm the existence of Cam OB3 as an association in the Cygnus arm and allow an accurate determination of its distance as $4.0 \pm 0.4 \mathrm{kpc}$.

Acknowledgements. We are very grateful to Jesús Maíz Apellániz for advice on the use and interpretation of the CHORIZOS code. We also thank the referee for helpful comments that improved the paper.

This research has been partially supported by the Ministerio de Educación y Ciencia under grant AYA2005-00095 and by the Generalitat Valenciana under grant GV04B/729.

The INT is operated on the island of La Palma by the Isaac Newton Group in the Spanish Observatorio del Roque de Los Muchachos of the Instituto de Astrofísica de Canarias. Based in part on observations made at Observatoire de Haute Provence (CNRS), France. The Nordic Optical Telescope is operated on the island of La Palma jointly by Denmark, Finland, Iceland, Norway, 
and Sweden, in the Spanish Observatorio del Roque de los Muchachos of the Instituto de Astrofisica de Canarias. The data were taken with ALFOSC, which is owned by the Instituto de Astrofísica de Andalucía (IAA) and operated at the Nordic Optical Telescope under agreement between IAA and the NBIfAFG of the Astronomical Observatory of Copenhagen.

This research has made use of the Simbad data base, operated at CDS, Strasbourg, France. This publication makes use of data products from the Two Micron All Sky Survey, which is a joint project of the University of Massachusetts and the Infrared Processing and Analysis Center/California Institute of Technology, funded by the National Aeronautics and Space Administration and the National Science Foundation.

\section{References}

Benjamin, R. A., Churchwell, E., Babler, B. L., et al. 2005, ApJ, 630, L149 Cardelli, J. A., Clayton, G. C., \& Mathis, J. S. 1989, ApJ, 345, 245

Crawford, D. L., Glaspey, J. W., \& Perry, C. L. 1970, AJ, 75, 822

Dame, T. M., Hartmann, D., \& Thaddeus, P. 2001, ApJ, 547, 792

Deharveng, L., Zavagno, A., Salas, L., et al. 2003, A\&A, 399, 1135

Draper, P. W., Taylor, M., \& Allan, A. 2000, Starlink User Note 139.12, R.A.L.

Fernández, D., Figueras, F., \& Torra, J. 2001, A\&A, 372, 833

Georgelin, Y. M., \& Georgelin, Y. P. 1976, A\&A, 49, 57

Haug, U. 1970, A\&AS, 1, 35

Hiltner, W. A. 1956, ApJS, 2, 389
Howarth, I., Murray, J., Mills, D., \& Berry, D. S. 1997, Starlink User Note 50.20, R.A.L.

Humphreys, R. M. 1978, ApJS, 38, 309

Humphreys, R. M., \& McElroy, D. B. 1984, ApJ, 284, 565

Kroupa, P. 2001, MNRAS, 322, 231

Leonard, P. J. T., \& Duncan, M. J. 1990, AJ, 99, 608

Maíz-Apellániz, J. 2004, PASP, 116, 859

Marco, A., \& Bernabeu, G. 2001, A\&A, 372, 477

Martins, F., Schaerer, D., \& Hillier, D. J. 2005, A\&A, 436, 1049

Moffat, A. F. J., Fitzgerald, M. P., \& Jackson, P. D. 1979, A\&AS, 38, 197

Negueruela, I. 2004, AN, 325, 380

Negueruela, I., \& Marco, A. 2003, A\&A, 406, 119 (Paper I)

Negueruela, I., Marco, A., Israel, G. L., \& Bernabeu, G. 2007, A\&A, 471, 485

Perry, C. L., Olsen, E. H., \& Crawford, D. L. 1987, PASP, 99, 1184

Pflamm-Altenburg, J., \& Kroupa, P. 2006, MNRAS, 373, 259

Quillen, A. C. 2002, AJ, 124, 924

Russeil, D. 2003, A\&A, 397, 133

Rubin, V. C. 1965, ApJ, 142, 934

Shortridge, K., Meyerdicks, H., Currie, M., et al. 1997, Starlink User Note 86.15, R.A.L.

Skrutskie, M. F., Cutri, R. M., Stiening, R., et al. 2006, AJ, 131, 1163

Stetson, P. B. 1987, PASP, 99, 191

Vallée, J. P. 2005, AJ, 130, 569

Zdanavičius, J., Zdanavičius, K., \& Straizys, V. 2005, BaltA, 14, 31 